\title{
Composing interactive systems by planning
}

\author{
Yoann Gabillon \\ Laboratoire d'Informatique de \\ Grenoble (LIG) \\ 385 rue de la Bibliothèque BP53 \\ F-38041 Grenoble cedex 9 \\ (+33) (0)4 76635687 \\ yoann.gabillon@imag.fr
}

\author{
Gaëlle Calvary \\ Laboratoire d'Informatique de \\ Grenoble (LIG) \\ 385 rue de la Bibliothèque BP53 \\ F-38041 Grenoble cedex 9 \\ (+33) (0)4 76514854 \\ gaelle.calvary@imag.fr
}

\author{
Humbert Fiorino \\ Laboratoire d'Informatique de \\ Grenoble (LIG) \\ 46 avenue Félix Viallet \\ F-38031 Grenoble cedex \\ (+33) (0)4 76574785 \\ humbert.fiorino@imag.fr
}

\begin{abstract}
User Interfaces (UIs) are mostly produced along a mental forward engineering process consisting in step by step transforming abstract descriptions into more concrete ones. Transformations make trade-offs between the context of use (<user, platform, environment $>$ ) and the usability properties that have been elicited as key. In ubiquitous computing, neither the context of use nor the user's objectives can be set at design time: they may opportunistically emerge with the arrival/departure of interaction resources and/or user's needs. As a result, there is a need for dynamically composing interactive systems. We explore multiagents planning for tackling the combinatory issue when sharing interaction resources among interactive systems and UI elements. The gateway between HCI and planning is performed using Model Driven Engineering (MDE). Experience shows that MDE is powerful for chaining domains together as well as for better understanding and improving domains languages.
\end{abstract}

\section{RESUME}

Aujourd'hui, une Interface Homme-Machine (IHM) est le fruit de transformations souvent mentales, rarement explicitées, appliquées à une représentation abstraite de l'IHM appelée modèle de tâches. Ces transformations font des compromis entre le contexte d'usage courant (<Utilisateur, Plate-forme, Environnement $>$ ) et les propriétés d'utilisabilité à satisfaire. En informatique ambiante, le contexte d'usage devient variable et imprévisible. Il est donc nécessaire de doter le système de capacité de raisonnement (modèles et transformations) pour lui permettre de conformer l'IHM à ce contexte dynamique. Notre étude explore la Planification pour la composition dynamique d'un système interactif conforme à un objectif utilisateur (un modèle de tâches partiel) et un contexte d'usage donnés. L'Ingénierie Dirigée par les Modèles (IDM) est utilisée comme passerelle entre les deux communautés IHM et Planification. L'article présente un premier prototype et montre les convergences et divergences entre

Permission to make digital or hard copies of all or part of this work for personal or classroom use is granted without fee provided that copies are not made or distributed for profit or commercial advantage and that copies bear this notice and the full citation on the first page. To copy otherwise, or republish, to post on servers or to redistribute to lists, requires prior specific permission and/or a fee.

UbiMob'08, May 28-30, 2008, Saint-Malo, France.

Copyright 2008 ACM 978-1-59593-980-7/08/05 ..\$5.00.
IHM et Planification révélées par l'effort d'alignement.

\section{Categories and Subject Descriptors}

H.5.2 [User Interfaces]: Ergonomics, Graphical user interfaces (GUI), Prototyping, User-centered design. I.2.9 [Robotics]: Workcell organization and planning.

\section{General Terms}

Design, Experimentation, Human Factors.

\section{Keywords}

Composition dynamique, Contexte d'Usage, IHM, Planification, IDM.

\section{INTRODUCTION}

En informatique ambiante, les objectifs de l'utilisateur peuvent émerger opportunément. Le contexte d'usage [2] (plate-forme, environnement) peut varier dynamiquement. Il devient nécessaire de savoir composer dynamiquement des systèmes interactifs qui : (1) remplissent les objectifs fonctionnels de l'utilisateur (« faire telle tâche »), (2) aient les bonnes propriétés du point de vue de l'interaction homme-machine (temps de calcul, présentation), (3) soient compatibles du contexte d'usage perçu.

Cet article explore les techniques de planification automatique de tâches (ici appelées planification) pour la composition dynamique de systèmes interactifs. La planification produit des plans exécutables pour un objectif et un état du monde donnés. Le plan est un ordonnancement d'actions ou d'opérateurs (par exemple, déplacer un cube). Traditionnellement, la planification est étudiée en robotique. Elle est ici appliquée de façon originale à l'ingénierie des Interfaces Homme-Machine (IHM).

L'étude est originale à plusieurs titres :

- beaucoup de travaux étudient la composition statique de systèmes interactifs (composition de services Web, composition d'IHM) mais aucun ne cible la composition dynamique ;

- classiquement, la planification résout des problèmes fonctionnels. Elle n'a encore jamais été confrontée aux exigences de l'interaction homme-machine (partage de tâches entre l'homme et la machine avec des boucles de contrôle telles que l'itération ; distribution de l'IHM sur 
les plates-formes disponibles; ergonomie du plan produit) ;

- la description des IHM du point de l'ergonomie est un défi ;

- l'alliance des deux communautés est rare.

L'article présente notre premier prototype MAPPING puis l'approche suivie. Il montre ensuite les convergences et divergences mises en évidence entre les domaines IHM et Planification.

\section{CAS D'ETUDE ET DEMONSTRATEUR MAPPING}

Le cas d'étude est celui d'un chercheur (Bob) inscrit à une conférence.

Bob arrive en retard à une session d'intérêt. Déçu, il reste dans le couloir, faute de place dans l'amphithéâtre. Il suit la présentation à distance grâce à un mur écran installé dans le couloir, mais au moment des questions il aimerait se manifester. Il décide alors d'utiliser le système MAPPING (Models, Artificial Perception and Planning for INteractive systems Generation) pour pouvoir poser sa question au conférencier. Il spécifie son objectif «I want to ask questions » en langage naturel dans une fenêtre dédiée (Figure 1).
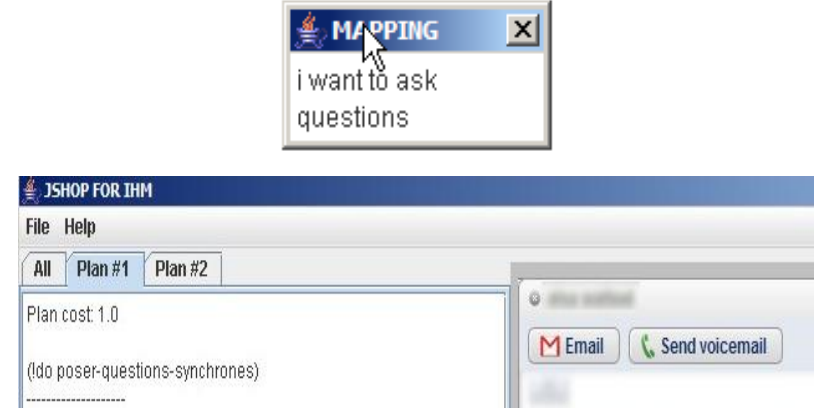

\begin{abstract}
Figure 1. Spécification de l'objectif utilisateur en langage naturel. Obtention de deux plans (\#1 et \#2) selon le mode synchrone ou asynchrone de la question.
\end{abstract}

Le système MAPPING analyse l'objectif utilisateur grâce au moteur de bon sens ConceptNet [5]. Il établit alors un modèle des tâches incomplet: dans l'exemple, il est réduit au seul objectif utilisateur («Poser des questions »). Aucune procédure (i.e., façon d'accomplir la tâche pour atteindre l'objectif) ne lui est associée. Grâce à un annuaire de services stockant des descriptions de systèmes interactifs en termes de tâche utilisateur offerte et de ressources d'interaction requises, MAPPING identifie deux procédures possibles pour atteindre le but : recourir à un outil de communication synchrone (par exemple, MSN) ou asynchrone (par exemple, le courrier électronique) (Figure 1). Les deux plans sont proposés à l'utilisateur.

La Figure 2 montre l'application de MAPPING à un exemple où la combinatoire est plus élevée: la tâche utilisateur est plus

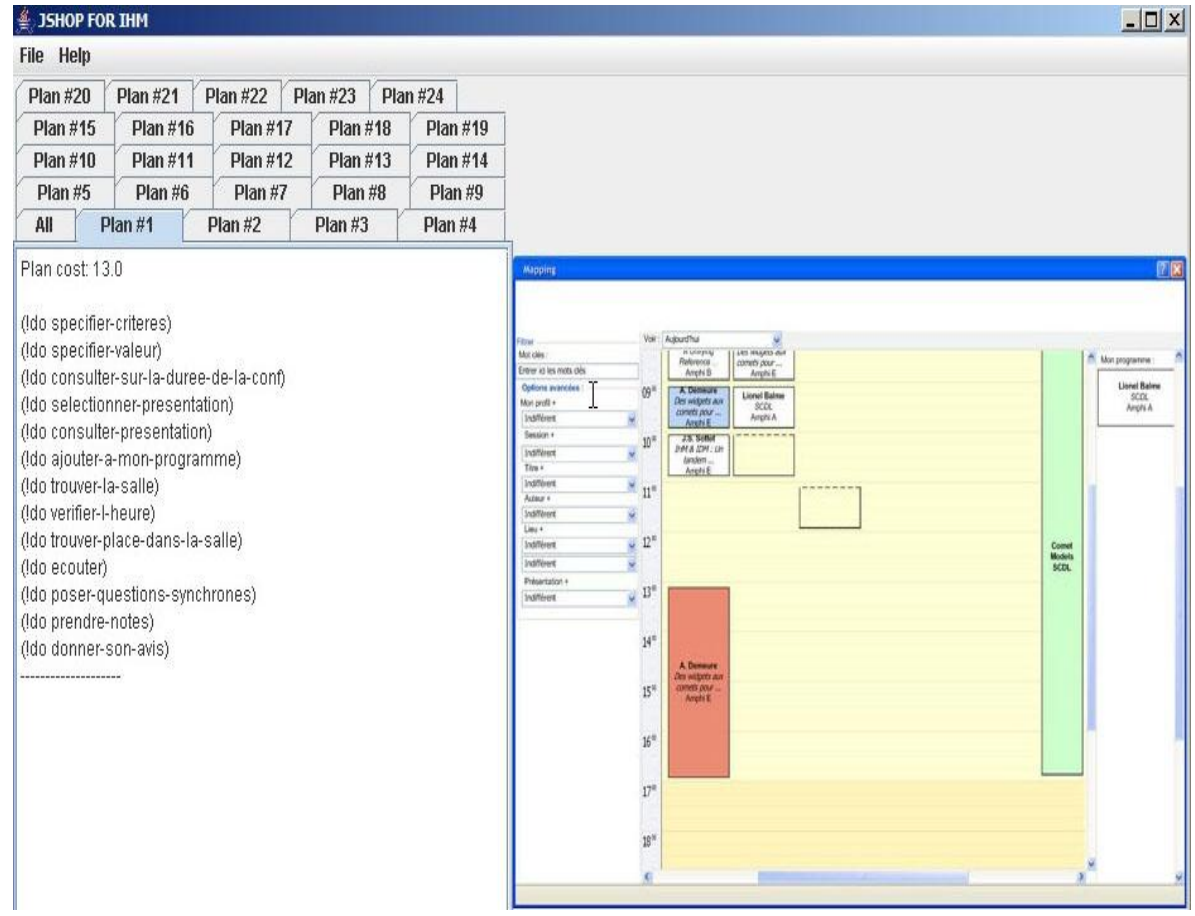

Figure 2. Vingt-quatre plans possibles pour un autre objectif. L'IHM du plan sélectionné (\#1) s'affiche en partie droite.

complexe (assister à des présentations sur un sujet donné) et la plate-forme plus variée : réduite à un seul PC en Figure 1, elle est ici étendue à un PC et un PDA, allumés ou éteints, branchés ou non à un clavier physique. L'utilisateur (anglophone) et l'environnement (sonore) restent fixés. Vingt-quatre plans sont trouvés et présentés à l'utilisateur.

\section{APPROCHE}

Le cas d'étude soulève deux difficultés :

1. Percevoir les exigences (l'objectif utilisateur et les propriétés d'utilisabilité attendues) et les ressources disponibles (dispositifs d'entrée et de sortie, unités de calcul, capacités de communication, composants réutilisables) ;

2. Calculer un Noyau Fonctionnel (NF) et une IHM conformes aux exigences et ressources.

Dans MAPPING, l'objectif utilisateur est formulé en langage naturel, par l'utilisateur lui-même. Les exigences d'utilisabilité ne sont pas formulées. L'ergonomie du plan produit n'est pas encore traitée. Les ressources sont déclarées par le concepteur au système. Le calcul se fait par planification: c'est l'approche 
suivie dans ce travail pour répondre au problème de la combinatoire.

L'Ingénierie Dirigée par les Modèles (IDM) est utilisée comme passerelle entre les communautés IHM et Planification. De façon plus précise, l'approche s'articule en cinq temps (Figure 3) :

1. Spécification du problème dans le domaine IHM : l'objectif utilisateur est analysé et reformulé en un modèle de tâches [3] [7] incomplet. Dans MAPPING, l'analyse se fait via ConceptNet [5].

2. Réécriture du problème dans le domaine de la Planification [6] : le modèle de tâches est transformé en un problème de planification ; les ressources définissent l'état du monde.

3. Calcul de plans par un planificateur : le calcul s'appuie sur les composants réutilisables (modèles ou code relevant du NF ou de l'IHM). Dans MAPPING, une IHM est associée à chaque modèle de tâche. Les modèles et codes sont entreposés dans un annuaire de services. Le planificateur est JSHOP [4]. JSHOP combine les modèles de tâches pour réaliser l'objectif utilisateur compte tenu des ressources disponibles. JSHOP renvoie un plan: une séquence d'actions physiques. JSHOP n'est pas un planificateur multiagents. Ce prototype est réalisé pour pratiquer et expliquer notre approche. Un planificateur multi-agents a les mêmes entrées/sorties.

4. Réécriture des plans en IHM : les plans sont reformulés en modèles de tâches.

5. Transformation en IHM pour construire une IHM ergonomique : le modèle de tâche peut être restructuré par ajout de tâches abstraites pour typiquement éviter des modèles plats (arbres de profondeur 1) qui aboutiraient à des IHM en séquence. Des concepts peuvent être factorisés pour un meilleur Guidage / Groupement-distinction entre items [1]; etc. Aujourd'hui, tous les plans sont restitués à l'utilisateur : chaque plan fait l'objet d'un onglet dédié (Figures 1 et 2).

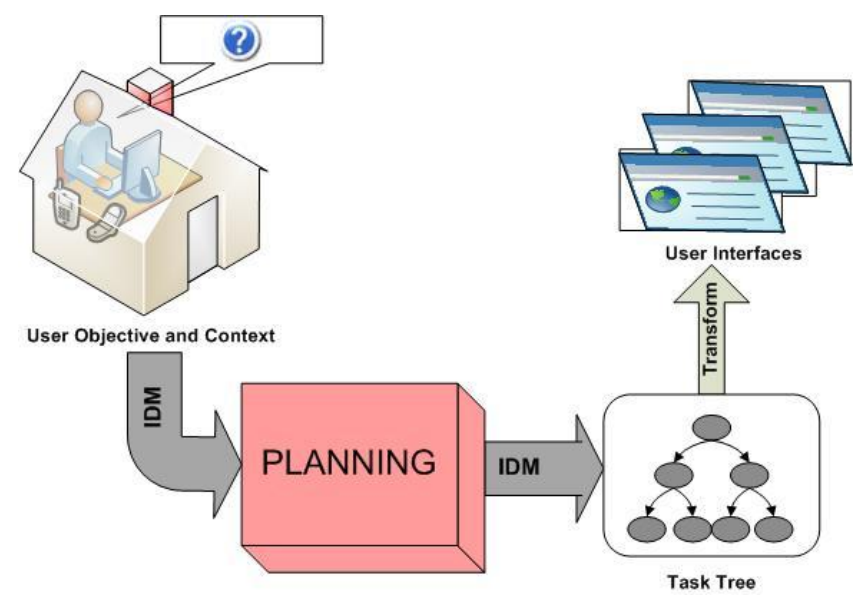

Figure 3. Approche globale.
MAPPING est un démonstrateur «en largeur ». De nombreuses questions restent ouvertes tant en description (quels langages de tâches, de ressources...) qu'en algorithmes (JSHOP, GraphPlan...). Aujourd'hui, notre travail s'est porté sur la décomposition fonctionnelle du problème, l'identification des verrous et la passerelle entre les deux communautés. Il a permis d'exhiber des convergences et divergences entre les deux communautés IHM et Planification.

\section{ALIGNEMENT ENTRE IHM ET PLANIFICATION}

Cette section établit les premières convergences et divergences entre IHM et Planification, pointées par l'alignement. La mise en correspondance pousse à une meilleure compréhension et formalisation de chaque domaine.

\subsection{Convergences}

La planification permet de résoudre un but. Pour cela, elle manipule une description de l'état du monde et un ensemble d'actions (opérateurs) possibles. La résolution du but s'exprime par un ensemble de plans décrits sous la forme d'une suite d'actions. Dans notre étude, l'objectif utilisateur (le but) est à résoudre dans un contexte d'usage (l'état du monde) variable en termes de ressources notamment. Mettre en correspondance les deux domaines IHM et Planification, équivaut à obtenir une expressivité équivalente entre les termes suivants :

- l'objectif utilisateur (communauté IHM) et le but à atteindre (communauté Planification) : en planification, le but à atteindre équivaut à un état du monde à atteindre, l'objectif utilisateur doit donc être exprimé de cette manière.

- le contexte d'usage (communauté IHM) et l'état du monde (communauté Planification) : la modélisation du contexte d'usage doit être exprimable de la même manière que l'état du monde, c'est-à-dire par des prédicats pouvant posséder des paramètres.

- un ensemble de services et un ensemble d'actions : un modèle de tâches (communauté IHM) représentant un service doit correspondre à une ou plusieurs actions (communauté Planification). Une action est composée de paramètres, de pré-conditions qui doivent être vraies pour pouvoir effectuer l'action, et de post-conditions qui modifient l'état du monde si l'action est réalisée.

- $\quad$ un ensemble d'IHM (communauté IHM) et un ensemble de plans (communauté Planification) : comme un plan correspond à une suite d'actions, un plan correspond d'après le point précédent - à un ensemble de services et donc, en conséquence, à un ensemble d'IHM.

Au-delà de ces convergences, des divergences apparaissent toutefois.

\subsection{Divergences}

La correspondance entre les deux communautés est loin d'être immédiate :

1) Divergences terminologiques: la notion d'opérateur, par exemple, est un faux ami. En IHM, il dénote les 
relations logiques ou temporelles entre tâches. En planification, il s'apparente à une fonction (par exemple, allumer(X) où $\mathrm{X}$ est une plate-forme).

2) Divergences sémantiques : le «ou» en IHM représente un choix pour l'utilisateur à proposer dans l'IHM finale tandis que le «ou» en planification réalise ce choix en ne sélectionnant dans un plan solution que l'une ou l'autre de ces actions. Les alternatives sont éliminées algorithmiquement.

3) Préoccupations différentes: dans son calcul, le planificateur connaît les tâches utilisateur abstraites mais ne les fournit pas en sortie. Il restitue une séquence d'actions physiques desquelles les tâches abstraites doivent être recalculées. Ces tâches abstraites sont en effet cruciales pour la construction d'IHM ergonomiques.

4) L'opérateur «entrelacement » qui permet à l'utilisateur de réaliser les mêmes tâches en même temps n'a pas d'équivalence en planification.

5) La décoration «itération » qui permet à une tâche d'être réalisée par l'utilisateur autant de fois qu'il le souhaite, pose le problème de récursivité infinie dans la construction du plan solution. Un planificateur ne trouvera pas de solution du fait de cette récursivité.

6) Les décorations de tâches «critique », «fréquente » et «optionnelle» ne sont actuellement pas exprimables dans un problème de planification.

Ces convergences et divergences entre domaines sont discutées dans le paragraphe suivant pour mettre en perspective les futurs travaux à réaliser.

\subsection{Discussion}

Dans une problématique de contexte variable et imprévisible, la planification présente par nature des atouts indéniables : parallèle entre les entrées (objectif utilisateur, contexte d'usage, actions) et les sorties (IHM permettant à l'utilisateur de réaliser son but) respectives; existence de planificateurs pour le calcul de procédures permettant de réaliser un but dans un état du monde donné. Cependant, la mise en correspondance est moins immédiate qu'il n'y parait et certaines différences d'expressivité posent de nouveaux verrous. Ces verrous appellent à une évolution des formalismes et algorithmes de planification pour répondre aux requis de l'interaction homme-machine. Ceci comprend, par exemple, la restitution des tâches abstraites en sortie des planificateurs ou encore l'intégration de boucles de contrôle telles que l'itération. Au-delà de ces évolutions, la mise en correspondance s'avère positive dans la meilleure compréhension des domaines et formalisation qu'elle suscite. Une différence fondamentale est ainsi apparue entre IHM et planification: alors qu'en IHM, un modèle de tâches peut contenir des alternatives proposées à l'utilisateur (le ou), en planification le choix entre alternatives est assumé par le système.

\section{CONCLUSION ET PERSPECTIVES}

L'article propose un parcours en largueur de notre approche pour la composition dynamique de systèmes interactifs. L'approche est illustrée sur un premier prototype MAPPING. Nous combinons deux domaines, l'IHM et la planification. L'expérience montre que l'effort de mise en correspondance est loin d'être stérile. Il force à une meilleure compréhension en sémantique et complétude de chaque domaine et permet de mettre en relief les difficultés d'une telle problématique. En perspective, nous ciblons un travail «en profondeur» sur les descriptions et l'algorithmique. MAPPING v2 fera l'objet d'évaluations pour comprendre le degré d'observabilité et de contrôle à donner à l'utilisateur dans le processus de composition.

\section{REMERCIEMENTS}

Nous remercions la Région Rhône-Alpes, via le cluster ISLE, projet PRESENCE pour le financement de ce travail.

\section{REFERENCES}

[1] Bastien JM., Scapin D. 1993. Ergonomic Criteria for the Evaluation of Human-Computer Interaction, Rapport Technique $\mathrm{n}^{\circ} 156$, juin 1993, INRIA.

[2] Florins M. 2006. Method for Designing Multiplatform Graphical User Interfaces, Thèse de doctorat, Université catholique de Louvain.

[3] Guilio M., Paterno F., Santoro C. 2004. Design and Development of Multidevice User Interfaces through Multiple Logical Description, IEEE Transactions on Software Engineering, vol. 20, $\mathrm{n}^{\circ}$ 8, p. 507-520.

[4] Ilghami O., Nau DS. 2003. A general approach to synthesize problem-specific planners, Technical report CS-TR-4597, UMIACS-TR-2004-40, University of Maryland, October 2003.

[5] Liu, H., Singh, P. 2004. Focusing on ConceptNet's natural language knowledge representation, Commonsense Reasoning in and over Natural Language Proceedings of the 8th International Conference on Knowledge-Based Intelligent Information \& Engineering Systems (Wellington, New Zealand, September 22 - 24, 2004) KES'2004, Lecture Notes in Artificial Intelligence.

[6] Nau, D., Ghallab, M., Traverso, P. 2004. Automated Planning: Theory and practice, Morgan Kaufmann Publishers Inc.

[7] Sottet JS., Calvary G., Favre JM. 2006. Towards mapping and model transformation for consistency of Plastic User Interfaces, Computer Human Interaction, Workshop on The Many Faces of Consistency in Cross-platform Design. 